\title{
Radial Distribution Function Analysis of the Polyamide Thin Film Composite formation using Trimesoyl Chloride and Piperazine
}

\author{
W.Z.A.W. Jusoh', S.A. Rahman ${ }^{1 *}$, A.L. Ahmad ${ }^{2}$ and N.M. Mokhtar ${ }^{3}$ \\ ${ }^{1}$ Faculty of Chemical and Natural Resources Engineering, Universiti Malaysia Pahang, 26300 Kuantan, \\ Pahang, Malaysia \\ ${ }^{2}$ Pusat Pengajian Kejuruteraan Kimia, Kampus Kejuruteraan, Universiti Sains Malaysia 11800 Universiti Sains \\ Malaysia Pulai Pinang, Malaysia \\ ${ }^{3}$ Faculty of Engineering Technology, Universiti Malaysia Pahang, 26300 Kuantan, Pahang, Malaysia \\ *Email: sunarti@ump.edu.my
}

\begin{abstract}
Polyamide thin film composite (TFC) membranes are well known for their performance and strength which normally applied in pervaporation dehydration and reverse osmosis field. TFC is produced by the rapid reaction between aqueous and organic monomer. However, the interaction between monomers is not well discussed at the atomic level and there is no precise tool to measure the effectiveness of the selection of organic with the aqueous monomer to form a stable TFC layer. Thus, this paper aims to analyze the interaction between aqueous monomer and organic monomer using the Molecular Dynamic (MD) simulation to form the TFC membrane. This work was done by using Piperazine (PIP) as the aqueous monomer with the combination of Trimesoyl Chloride (TMC) as the organic monomer in the binary systems. The simulation involved the setting of Ewald Summation Method, COMPASS force field, equilibrium phase by microcanonical, NVE (constant volumes and total energy) and run-production stage by NPT (constant pressure and temperature). Analysis by the Radial distribution function (RDF) explicates the intermolecular interfacial of $5.75 \AA$ between the bonding of $\mathrm{N}$ (Amine) - C (TMC) atoms at the distance of $1.0 \AA$. This study suggested that the TFC formed by the interaction between TMC - PIP is very much stable based on the higher interaction in a very short distance.
\end{abstract}

Keywords- Aqueous Monomers; COMPASS Force Field; Dynamic Molecular; Interfacial Polymerization; Thin Film Composite Membrane

\section{INTRODUCTION}

Thin film composite (TFC) is generally produced by a rapid interfacial polymerization (IP) reaction between amine and acyl chloride that represents aqueous monomer and organic monomer, respectively. Prompt formation of a thin polyamide layer is expected to form when the acyl chloride-based monomer gets contact with the amine-based monomer at the bi-solution interface. This IP method promotes the production of a thin selective layer on top of the substrate which directly contributes to the higher permeate flux and good selectivity. The addition of the thin film layer would not affect the substrate structure as the substrate only acts as the mechanical support for the TFC membrane and does not interfere with the mass transport process.

TFC is considered one of the modification methods on the dense membrane structure and commonly used in the previous researches. This is because the typical dense membrane is easily wetted during separation processes which shorten the exposure time when contacting with the liquid in the pervaporation (PV), nano-filtration (NF), reverse osmosis (RO) and other processes. To avert such flaws with an effective and economical way, TFC is the ideal choice to enclose a thin layer of hydrophilic polymer on top of the substrate produced by the IP method [1-3]. Zuo et al. applied the interfacial 
polymerization technique to the TFC membranes with m-phenylenediamine (MPD) and hyperbranched polyethyleneimine (HPEI) with the trimesoyl chloride (TMC) [4,5]. In some cases, TFC membranes were found did not enhance the separation factor performances. However, there were few studies found that utilizing the TFC did contribute to the major escalation in the separation factor [6,7]. This may due to the loose TFC membrane formed and swipe out during operation. Choi et al. stressed that in the end, the importance of the fundamental study of membrane structure-performance relationship to development better TFC membranes [8]. Thus, in addition to the parameter for the interfacial polymerization mechanism, it is intrinsically to have an accurate selection of monomers.

Molecular dynamics (MD) simulation is purposely used to simulate the interaction between the molecular structures at the atomistic level. It is widely used in the chemical process and pharmaceutical field. The study on the compatibility of the materials blended is reported by researchers on polyvinylmethylether/polystyrene, polymers/carbon nanotubes and Poly (vinyl alcohol) and Chitosan using COMPASS force field setting [9-11]. Moreover, the system process and error of the actual process can be firstly estimated using the simulation which assists to reduce the time and cost [12]. MD is known for its accurate data which compatible with the results obtained from the actual process [1315].

Any defects and international stresses on the surfaces could deteriorate the membrane mechanical strength. As one of the simple and economic alternative, IP provides a good strategy to overcome this limitation by TFC polymer matrix. Thus it is very important, to ensure a good interfacial binding for improving the polymer function and properties to be applied to processes. However, the strength of the interfacial binding very dependable on the selection type of the aqueous - organic monomers. In our simulations study, we focus on the chemical interaction between TMC and organic monomers specifically Piperazine (PIP). For that reason, molecular dynamics simulations with the COMPASS force field were the best for examining intermolecular bonding during the IP process.

\section{MODEL VALIDATION}

\subsection{Monomers selection using Molecular Dynamic}

The membrane simulations were applied to define the interaction of organic and aqueous monomers. In this study, amines PIP were chosen to undergo IP reaction with TMC to produce the TFC membrane as shown in Table 1. TMC is considered superior as organic solvents and applied in the most TFC membrane $[2,3$, and 16$]$.

Table 1: Density of TMC-aqueous monomers

\begin{tabular}{lll}
\hline Monomers & Name & Density $(\mathrm{g} / \mathrm{mol})$ \\
\hline Organic & TMC & 265.47 \\
Aqueous & PIP & 86.14 \\
\hline
\end{tabular}

The best pair of organic and aqueous monomer was analysed by radial distribution function (RDF) analysis in the MD for the shortest distance for the interaction happen as well as the magnitude of the attraction forces intra-molecules. The strongest bonding purposed the best depositing of PA TFC on the subtract membrane. The common molecular structures of the monomers with the different number of functional groups and chain length were presented in Figure 1. Generally, TMC having three acyl chloride groups attach to a benzene ring and PIP contains two amine groups to create the polyamide with the crosslinking structure. 
(a)

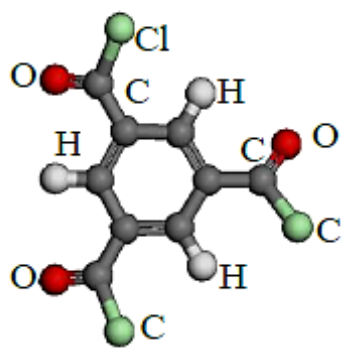

TMC (b)

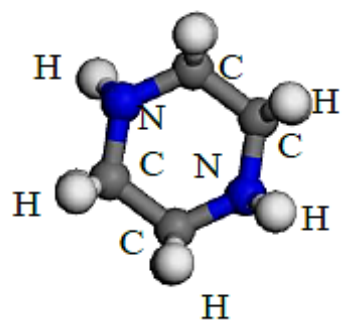

PIP

Figure 1: The repeat unit in the monomers chain molecule: (a) TMC and (b) PIP.

\subsection{Molecular Dynamic simulations on the Interfacial Polymerization Process}

MD simulation study of crosslinking between aqueous monomer-TMC had been performed using Material Studio version 7.0 software provided by Accelrys. The simulation involved the setting of Ewald Summation Method and COMPASS force field. The equilibrium phase was performing in the microcanonical, NVE (number molecules, volumes, and total energy) followed by run-production stage by NPT (number molecules, pressure, and temperature).

The computer simulations have been performed in three dimensions [17] for an MD cell of a volume $\mathrm{V}=(22.810 \times 22.812 \times 22.814) \AA^{3}$ under both the energy and temperature control ensembles at $\mathrm{T}=298$ $\mathrm{K}$ as shown in Figure 2. Starting with a 10:10 (by molecules) TMC-aqueous monomer solution, with a corresponding density of about $1.00 \mathrm{~g} / \mathrm{cm}^{3}$. The simulations were run for a 1000 picoseconds (ps) with the time step set 1 femtosecond (fs) for each NVE and NPT runs. The magnitude of the attraction or repulsion interaction between PIP - TMC was analysed by Radial distribution function analysis (RDF).

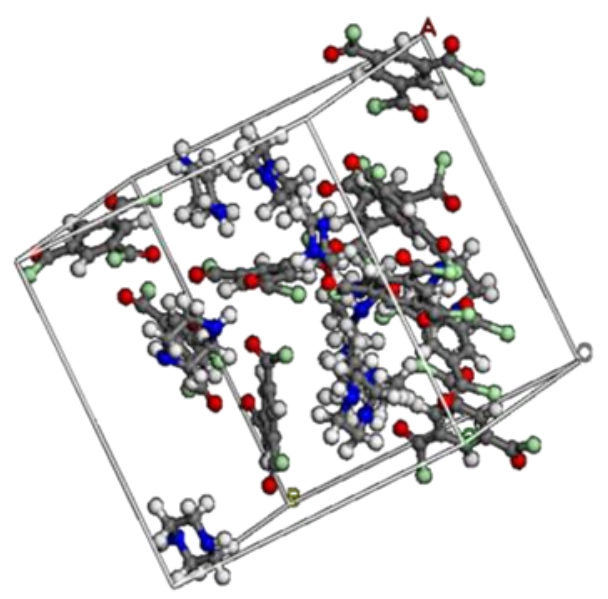

Figure 2: 3D box represent the simulation of crosslinking of 10:10 (a) TMC-PIP in a binary system with the dimensions. 


\section{RESULT AND DISCUSSIONS}

\subsection{Final cross-linking density}

The final cross-linking density of the TFC membranes was computed in the run-production stage by NPT ensembles for $1000 \mathrm{ps}$ was found about 1.337. This result is in good agreement with the most simulation works and varies from 1.1-1.38 g/cm3 [18-20]. These validations are acceptable to support the force field parameters run in these MD simulations. According to Huang et al., higher the crosslinking density acquired by the shorter aqueous monomers structure [6]. It was revealed in the simulation of the shortest molecular chain, pointed out the highest cross-linking density. In the meantime, a lower degree of crosslinking yield higher polymerization rate [21].

\subsection{Cross-linking between acyl and amine functional group}

Cross-linking is a process of announcing of bonding connection between a component to the preexisting polymer to form a large and complex repeating unit of polymer molecules. All network chains have reacted on both ends, and each end of all chains is attached to the network at different junction points. There are two main interaction forces involved for cross-link to fabricate TFC membrane which are intra-molecular and intermolecular interaction. These forces can be identified using RDF analysis from the MD simulations. Intermolecular is the major attractive forces among the atoms from different molecules nearby to form larger molecules structure. First, Hydrogen $(\mathrm{H})$ atoms leaving amine main chain form a covalent bond with Chloride $(\mathrm{Cl})$ atoms which drawn from TMC molecule to form byproduct of $\mathrm{HCl}$ then Nitrogen $(\mathrm{N})$ from amine have attractive interact with the Carbon $(\mathrm{C})$ from acyl group as shown in Figure 3. The bonding was assigned by the Van der Waal's (VDW) parameters. While intra-molecular is considered as minor atoms interaction. Basically, intra-molecular is the repulsive forces between atoms with their adjacent atom in a molecule.

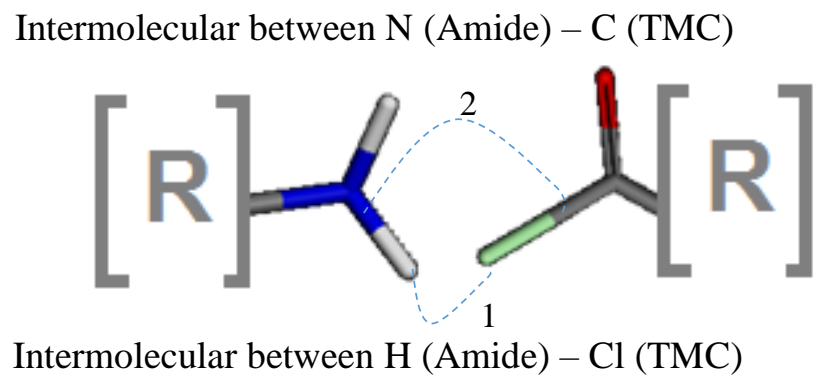

Figure 3: Intermolecular forces existed in the binary system of TMC - PIP, where 1) intermolecular bond between $\mathrm{H}$ from amide group with $\mathrm{Cl}$ from acryl group, 2) intermolecular bond between $\mathrm{N}$ from amide group with $\mathrm{C}$ from acryl group

\subsection{RDFs analysis of the intermolecular interaction}

Referring to Figure 4, the main correlations to form a polymeric network is the bonding between $\mathrm{C}$ atom TMC molecule and Nitrogen $\mathrm{N}$ atom from the amine molecule. Results show that the most preferential sites of interactions are the between the $\mathrm{C}$ atom in TMC with the $\mathrm{N}$ atom from monomers (maximum of the first peak). The trend shows that the distance between $\mathrm{N}$ (amine) - C (TMC) atoms are in the range of $5.75 \AA$ to start the intermolecular interaction with the intensity between $1.0 \AA$ with each other. Higher the density of chain packing donated to the larger transformation in the mean-square bond length and mean-square bond angle which contributed to rising the bond stretching and bond bending inside the chain [22]. The factors significantly elaborate on the enhancement of the intermolecular interaction as well. 


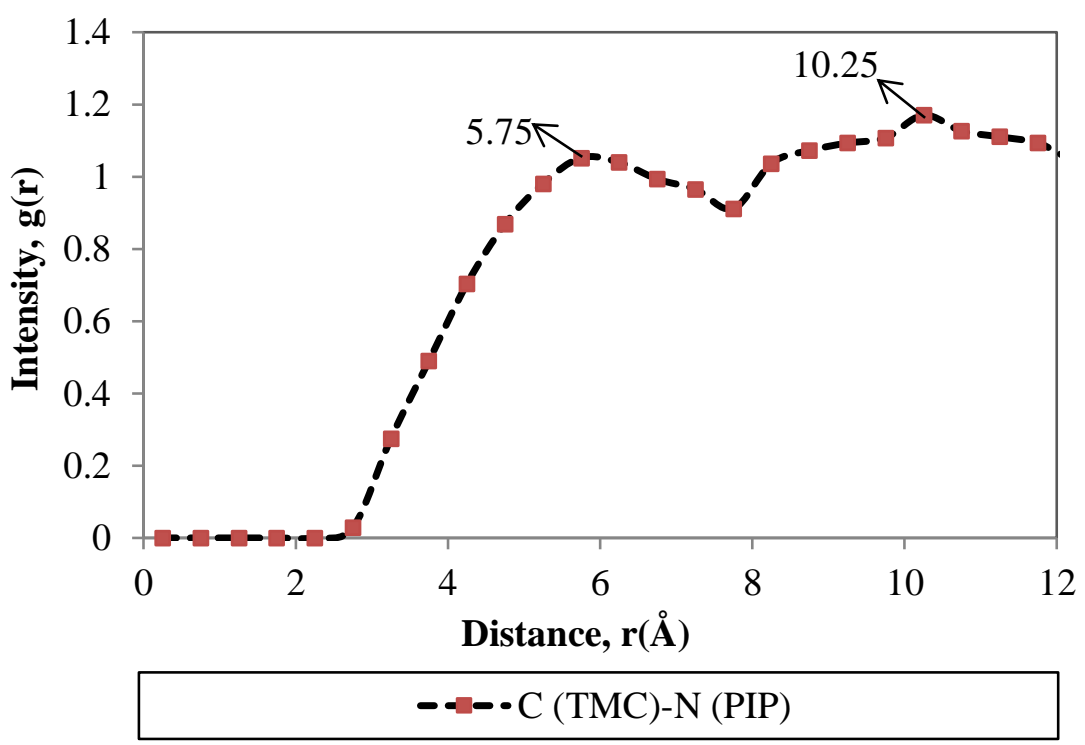

Figure 4: Inter-molecular attractions between Carbon atoms of TMC and Nitrogen of PIP at 298K

The finding is considered lower compared with the work by Shen et al., which attained the $\mathrm{C}-\mathrm{N}$ atoms binding at the distance of $3.5 \AA$ between TMC and m-phenylenediamine respectively using AMBER Force Field (GAFF) in MD simulation. This can be described by considering the structure of the PIP [23]. PIP reported to have weakest reaction rate during the interfacial polymerization may due to the steric hindrance effect. The results obtained agreed with the previous work carried by Huang et al.,[6]. Existing of the steric bulk effected the IP reaction to become much slower. However, the binding between TMC - PIP is still considered stable as the interaction occurred within the $10 \AA$ distance.

Monomer affinity with each other is an important characteristic to generate a sufficiently thin TFC layer but excellent perm-selective membranes performance. Both repulsive and attractive forces presented in the molecules are strongest which also attributed to the thin layer within the nanoscale which very desirable for solvent-solvent separation relatively [24]. RDFs Trend described that the PIP owned the alicyclic ring still able to form more stiff TFC molecular chain however TMC-PIP system only produced semi-aromatic chain [25]. In the PIP-TMC interaction, there were two significant peaks which at the 5.75 and $10.25 \AA$ with an average intensity of $1.11 \AA$ suggested that longer time step required. This also can include that the polymerization rate of this binary system is weak. The stable and compact TFC membrane is consequently reflected the membrane performances.

TFC membrane chain packing density was significantly related to the IP reaction rate. Furthermore, according to Tsai et al., (2017) membrane created by lower chain density can be less rigid due to penetration of aqueous monomers into TMC solution during reaction process compared with an aqueous monomer which has greater molecule size monomers which resulting to the poor selectivity result[26].

\section{CONCLUSIONS}

The idea to form a compact TFC structure on the support membrane is to avoid the loose TFC membrane and wash away when contacting with the feed solutions. The stability of the aqueous -organic monomers interfacial polymerization reaction was examined by the intermolecular atomic interaction analysis. RDFs analysis shows the main bond between $\mathrm{N}$-atoms in PIP and C-atoms in TMC within the distance of less than $10 \AA$. The high intensity at this short distance indicates that the structures of cross- 
linked PA formed are compact and stable. The RDFs analysis also shows that the interaction between TMC with PIP depending on the cross-linking density and monomers molecule size.

\section{ACKNOWLEDGEMENT}

The authors thank the sponsor for financial support via the grant of PGRS180323.

\section{REFERENCES}

[1] Ong, Y. T., and. Tan S. H., "Pervaporation separation of a ternary azeotrope containing ethyl acetate, ethanol and water using a buckypaper supported ionic liquid membrane," Chem. Eng. Res. Des., vol. 109, pp. 116-126, 2016.

[2] Shi, G. M., and. Chung, T. S., "Thin film composite membranes on ceramic for pervaporation dehydration of isopropanol," J. Memb. Sci., vol. 448, pp. 34-43, 2013.

[3] Zuo, J., Wang, Y., Sun, S. P., and Chung, T. S., "Molecular design of thin film composite (TFC) hollow fiber membranes for isopropanol dehydration via pervaporation," J. Memb. Sci., vol. 405406, pp. 123-133, 2012.

[4] Albo, J., Wang, J., and Tsuru, T., "Application of interfacially polymerized polyamide composite membranes to isopropanol dehydration: Effect of membrane pre-treatment and temperature," $J$. Memb. Sci., vol. 453, pp. 384-393, 2014.

[5] Tsuru, T., Sasaki, S., Kamada, T., Shintani, T., Ohara, T., Nagasawa, H., Nishida, K., Kanezashi, M., Yoshioka, T., "Multilayered polyamide membranes by spray-assisted 2-step interfacial polymerization for increased performance of trimesoyl chloride (TMC)/m-phenylenediamine (MPD)-derived polyamide membranes," J. Memb. Sci., vol. 446, pp. 504-512, 2013.

[6] Huang, S. H., Hsu, C. J., Liaw, D. J., Hu, C. C., Lee, K. R. and Lai, J. Y., "Effect of chemical structures of amines on physicochemical properties of active layers and dehydration of isopropanol through interfacially polymerized thin-film composite membranes," J. Memb. Sci., vol. 307, no. 1, pp. 73-81, 2008.

[7] Zhang, Y., Le, N. L., Chung, T. S., and Wang, Y., "Thin-film composite membranes with modified polyvinylidene fluoride substrate for ethanol dehydration via pervaporation," Chem. Eng. Sci., vol. 118, pp. 173-183, 2014.

[8] Choi, W., Jeona, S., Kwon, S.J., Park, H., Park, Y-I., Nam, S-E, Lee, P.S., Lee, J.S., Choi, J., Hong,S., Chane, E. P., Lee, J-H., "Thin film composite reverse osmosis membranes prepared via layered interfacial polymerization," J. Memb. Sci., vol. 527, no. December 2016, pp. 121-128, 2017.

[9] Ahmadi, A., and Freire, J. J., "Molecular dynamics simulation study of compatibility for the polyvinylmethylether/polystyrene mixture," Mol. Simul., vol. 34, no. 10-15, pp. 1253-1258, 2008.

[10] Yang, M., Koutsos, V., and Zaiser, M., "Interactions between polymers and carbon nanotubes: A molecular dynamics study," J. Phys. Chem. B, vol. 109, no. 20, pp. 10009-10014, 2005.

[11] Jawalkar, S. S., Raju, K. V. S. N., Halligudi, S. B., Sairam, M., and Aminabhavi, T. M., "Molecular modeling simulations to predict compatibility of poly(vinyl alcohol) and chitosan blends: A comparison with experiments," J. Phys. Chem. B, vol. 111, no. 10, pp. 2431-2439, 2007.

[12] Kolev, V., and Freger, V., "Hydration, porosity and water dynamics in the polyamide layer of reverse osmosis membranes: A molecular dynamics study," Polym. (United Kingdom), vol. 55, no. 6, pp. 1420-1426, 2014.

[13] Meddah, C., Milchev, A., Sabeur, S. A., and Skvortsov, A. M., "Molecular weight effects on interfacial properties of linear and ring polymer melts: A molecular dynamics study," J. Chem. Phys., vol. 145, no. 19, 2016.

[14] Kholmurodov, K., Dushanov, E., Yasuoka, K., Khalil, H., Galal, A., Ahmed, S., Sweilam, N., and Moharram, H., "Molecular dynamics simulation of the interaction of ethanol-water mixture with a Pt surface," Nat. Sci., vol. 3, no. 12, pp. 1011-1021, 2011. 
[15] González, M. A., "Force fields and molecular dynamics simulations," Collect. SFN, vol. 12, pp. 169-200, 2011.

[16] Khorshidi, B., Soltannia, B., Thundat, T., and Sadrzadeh, M., "Synthesis of thin film composite polyamide membranes: Effect of monohydric and polyhydric alcohol additives in aqueous solution," J. Memb. Sci., vol. 523, pp. 336-345, 2017.

[17] Li, M., Liu, X. Y., Qin, J. Q., and Gu, Y., "Molecular dynamics simulation on glass transition temperature of isomeric polyimide," Express Polym. Lett., vol. 3, no. 10, pp. 665-675, 2009.

[18] Ding, M., Szymczyk, A., Goujon, F., Soldera, A., and Ghoufi, A., "Structure and dynamics of water confined in a polyamide reverse-osmosis membrane: A molecular-simulation study," $J$. Memb. Sci., vol. 458, pp. 236-244, 2014.

[19] Shen, M., Keten, S., and Lueptow, R. M., "Dynamics of water and solute transport in polymeric reverse osmosis membranes via molecular dynamics simulations," J. Memb. Sci., vol. 506, pp. 95 108, 2016.

[20] Xiang, Y., Liu, Y., Mi, B., and Leng, Y., "Hydrated polyamide membrane and its interaction with alginate: A molecular dynamics study," Langmuir, vol. 29, no. 37, pp. 11600-11608, 2013.

[21] Hutchins, K. M., Lee, C. Y. , Luo, B. , Chen, Q., and Moore, J. S., "Effects of Cross-Linking Density on Interfacial Polymerization and Scaffold Formation in Functionalized Polymer Beads," Ind. Eng. Chem. Res., vol. 56, no. 16, pp. 4883-4886, 2017.

[22] Zhao, J., Yu, P., and Dong, S., "The influence of crosslink density on the failure behavior in amorphous polymers by molecular dynamics simulations," Materials (Basel)., vol. 9, no. 4, p. 234, 2016.

[23] Shen, M., Keten, S., and Lueptow, R. M., "Rejection mechanisms for contaminants in polyamide reverse osmosis membranes," J. Memb. Sci., vol. 509, pp. 36-47, 2016.

[24] Ridgway, H. F., Orbell, J., and Gray, S., "Molecular simulations of polyamide membrane materials used in desalination and water reuse applications: Recent developments and future prospects," $J$. Memb. Sci., vol. 524, pp. 436-448, 2017.

[25] Tang, C. Y., Kwon, Y. N., and Leckie, J. O., "Effect of membrane chemistry and coating layer on physiochemical properties of thin film composite polyamide RO and NF membranes. I. FTIR and XPS characterization of polyamide and coating layer chemistry," Desalination, vol. 242, no. 1-3, pp. 149-167, 2009.

[26] Tsai, H. A., Wang, T-Y., Huang, S-H., Hu, C-C., Hung, W-S., Lee, K-R., and Lai, J-Y., "The preparation of polyamide/polyacrylonitrile thin film composite hollow fiber membranes for dehydration of ethanol mixtures," Sep. Purif. Technol., vol. 187, pp. 221-232, 2017. 